\title{
NFC Aplicado a Sistemas de Controle de Entrada em Eventos
}

\author{
Jairo Rodrigo Tomaszewski ${ }^{1}$, Daniel Formolo ${ }^{1}$ \\ ${ }^{1}$ Programa de Pós-Graduação em Desenvolvimento de Aplicativos para Dispositivos \\ Móveis - Universidade do Vale do Rio dos Sinos (UNISINOS) \\ Caixa Postal 15.064 - 91.501-970 - Porto Alegre - RS - Brasil \\ jairorodrigo@hotmail.com, danielformolo@unisinos.br
}

\begin{abstract}
This paper discusses current initiatives using NFC technology as substitute of tickets. The objective of this work is to propose a simple and feasible system to control the entering in events, using NFC technology present in smartphones, and evaluate the acceptance of such systems by people. The paper presents the architecture of the developed system and the evaluation of its use. The results show that NFC technology applied to ticket control has good acceptance and is promising.

Resumo. Este trabalho discute iniciativas atuais de uso da tecnologia NFC como substituta dos ingressos tradicionais. O objetivo deste trabalho é propor um sistema simples e viável de controle de entrada em eventos com uso da tecnologia NFC presente em smartphones e avaliar a aceitação de sistemas desse tipo junto à sociedade. O trabalho apresenta a arquitetura do sistema desenvolvido e avaliação de seu uso. Os resultados mostram que a tecnologia NFC aplicada no controle de ingressos tem boa aprovação e é promissora.
\end{abstract}

\section{Introdução}

Em um evento: festas, solenidades, shows, cerimônias, encontros ou jogos, sempre existe a necessidade de controlar a quantidade de pessoas que irão comparecer a ele, este é um fator primordial para sua organização. Segundo [Matos, 2012], o dinamismo das vendas relacionadas a um evento no menor tempo possível, é de extrema importância para o seu sucesso. O processo inicia com a disponibilização dos ingressos para a venda e continua até a acomodação do público no local do evento [Cesca, 2008].Entre essas etapas existe a entrada no local do evento, que atualmente é controlada com uso de ingressos tradicionais, geralmente impressos ou cartões. Por outro lado, o público que vai a eventos geralmente carrega consigo telefones celulares ou smartphones para registrar o evento e mesmo por segurança para o caso de alguma eventualidade que exija a comunicação com outras pessoas ou ainda por simples hábito.

$\mathrm{O}$ acesso a telefones celulares tem crescido a cada dia. A venda de smartphones no mundo tem crescido de forma acelerada. A Figura 1 mostra o crescimento das vendas de smartphones ao longo dos últimos anos. No ano de 2013 foram vendidos mais de 1 bilhão de unidades no mundo [IDC, 2014]. A previsão em 2018, é alcançar 1,7 bilhões de unidades vendidas [Teleco, 2015]. 


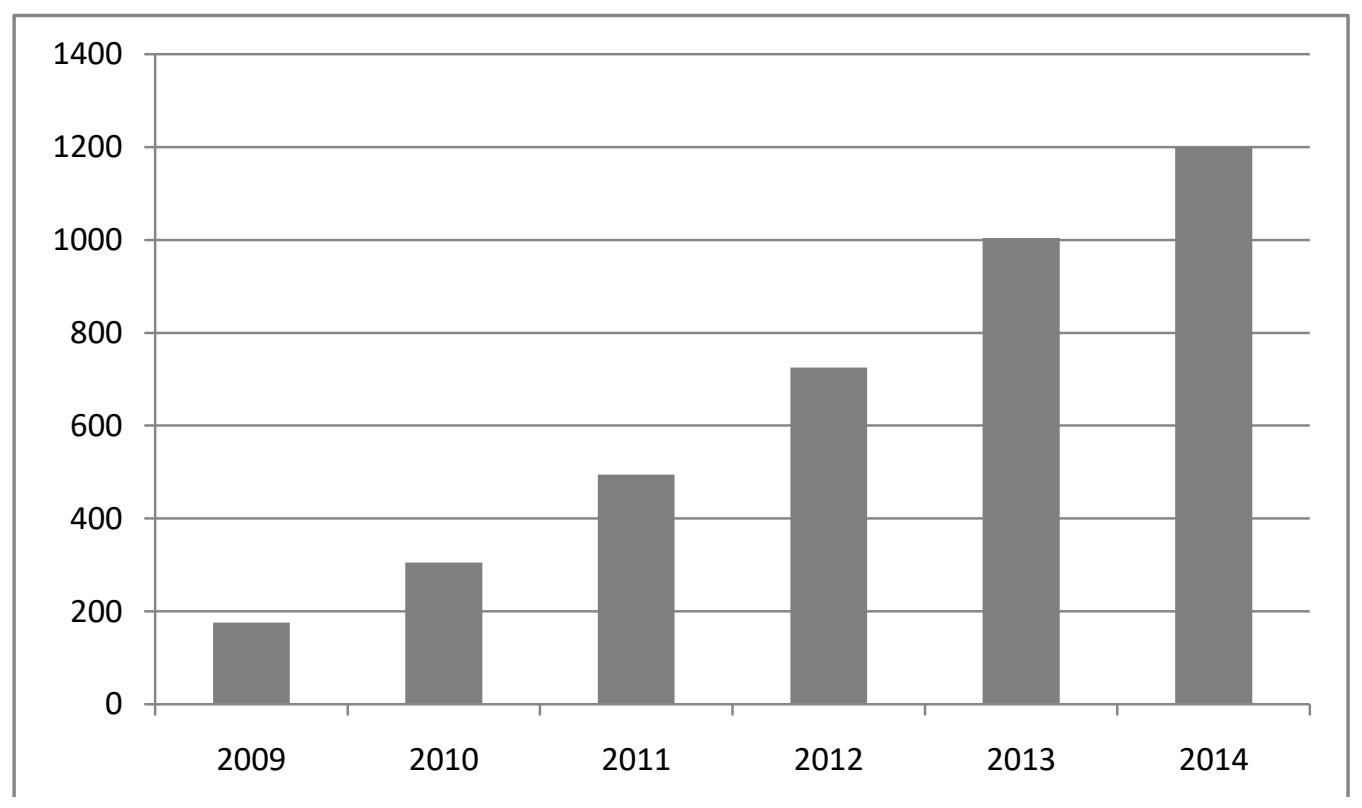

Figura 1. Vendas de smartphones no mundo em milhões de unidades [IDC, 2014], [Teleco, 2015].

Analisando este rápido crescimento pressupõe-se que sua popularização é inevitável, justificando qualquer solução que venha a usá-los como ferramenta de apoio em sistemas de software. Presume-se que o hábito de as pessoas utilizarem o smartphone na maior parte das atividades diárias [Oulasvirta, 2012], também se estende a atividades como eventos. Esse hábito pode ser explorado com o desenvolvimento de sistemas de acesso que utilizem smartphones, melhorando o controle e facilitando o acesso das pessoas aos eventos.

Além de telefone, conexão a redes e aplicativos, os smartphones disponibilizam sensores que ampliam a gama de aplicações que podem ser exploradas, [Boulos, 2011] identifica várias aplicações voltadas para saúde humana, [Keally, 2011] propõe um sistema para identificar a atividade que está sendo realizada pela pessoa portadora do smartphone, assim como esses exemplos, muitas outras aplicações vêm surgindo.Entre os sensores disponíveis nos smartphones um dos mais úteis nesse tipo de aplicação é o NFC (Near Field Communication), cuja tecnologia permite a troca de informações entre dois dispositivos sem contato através de radiofreqüência [Igoe, 2014]. Suas principais vantagens são a troca de dados facilitada entre dispositivos equipados com essa tecnologia e a segurança dessas transações [Michahelles, 2007]. Essas duas características têm uso em diversas aplicações do cotidiano, como a simples troca de imagens, contatos, informações em geral, carteira digital e identificador de identidade.

Em alguns países, já existem ensaios de uso de smartphones equipados com NFC como substituto de ingressos no controle de entrada em eventos, como os apresentados em [Keferi, 2012; Nasution, 2012; Boden, 2014]. Não existe um framework claro de qual é a melhor forma de implantar o NFC nesse contexto. Todas as propostas de uso têm variações que atendem aos hábitos da população local. Este trabalho propõe um novo modelo de uso de NFC aplicado a controle de ingressos em eventos e faz um estudo avaliando a aceitação do uso dessa tecnologia aplicada ao controle de ingressos no Brasil. 
A Seção 2 descreve brevemente o uso atual de ingressos. A Seção 3 detalha a tecnologia NFC, enquanto que a Seção 4 descreve modelos atuais de uso de NFC aplicados a controle de ingressos. A Seção 5 descreve o modelo proposto e implementado. A Seção 6 apresenta os resultados dos testes de usabilidade que foram aplicados sobre o sistema proposto, por fim a Seção 7 apresenta as conclusões do estudo.

\section{Sistemas de Controle de Acesso a Eventos}

A necessidade de controle de acesso a eventos sempre existiu, portanto, todo o processo desde a aquisição do ingresso até a entrada no evento é bem conhecido. Esse controle vem evoluindo ao longo do tempo. O uso de smartphones e NFC no controle de acesso a eventos é uma nova peça nessa inovação. Por ser relativamente novo, inclusive em outras áreas de aplicação, assume-se que a maioria dos usuários são pouco experientes no uso dessa tecnologia. Conforme Galitz (2007), quando os usuários não estão familiarizados com processos ou tecnologias empregadas, deve-se simplificar ao máximo as etapas de uso do sistema, limitando o número de possibilidades e informações de retorno sobre cada passo executado pelo usuário no sistema. Hix (1993) vai além, informando que a simplicidade no processo deve ser buscada em todos os sistemas. Tradicionalmente dois métodos de controle são utilizados, a seguir eles são descritos.

\subsection{Ingressos em Papel}

Ingresso em papel é a forma mais convencional e antiga utilizada pelos organizadores de eventos. Sua utilização requer um nível de trabalho razoavelmente grande, que compreende a definição da quantidade disponibilizada, contratação de uma empresa para fazer a impressão e realizar a venda em algum ponto físico. No momento do evento, deve-se receber cada pessoa e conferir a legitimidade de cada ingresso o que, por vezes, pode não ser eficiente devido à quantidade de pessoas acessando o local ao mesmo tempo.

Uma das grandes preocupações na utilização de ingressos em papel é sua falsificação, facilitada pelas tecnologias de impressão de alta qualidade cada vez mais acessíveis. Para evitar tal problema, a indústria de confecção de ingressos tem investido em materiais e tecnologias mais avançadas, o que dificulta a fraude, mas ao mesmo tempo encarece o produto. Um exemplo é a utilização de papel-dinheiro aliado a alguns outros elementos, citados a seguir.

- Holografia de segurança: chamado de EODV (Elemento Óptico Difrativo Variável) principal elemento de segurança. A holografia possui alterações de estrutura e cor.

- Rosácea ou Guilhoche: tramas em linhas contínuas entrelaçados em padrões geométricos positivos ou negativos.

- Fundo Numismático: aplicação em qualquer logo, linhas distorcidas de cor única ou multicolorida. Aparência de relevo latente ou 3D.

- Tinta luminescente: reagente à luz Ultravioleta ou raspagem de metal, em várias cores. 
Ainda, para evitar falsificações ou cópia de ingressos legítimos, são utilizados elementos adicionais, como códigos QR. Desta forma, cada ingresso pode conter mais informações como dados do comprador de forma criptografada [Gavinho, 2013].

Mesmo com todas essas tecnologias, ainda é necessária uma forma de verificação da sua legitimidade no momento da entrada do evento, o que também requer mais investimentos em pessoal e equipamentos sofisticados. Esse custo extra acaba por encarecer o preço do ingresso pago pelo usuário.

\subsection{Cartões}

Outro tipo de controle de entrada utilizado atualmente são os ingressos em cartão, normalmente fabricados em PVC. Tais cartões são mais difíceis de serem falsificados, além disto, podem conter uma tarja magnética para aumentar a segurança [Souza, 2013].

Algumas entidades, como clubes de futebol, fornecem um cartão ao seu associado que pode simplesmente ser recarregado via Internet para assistir a um evento. No entanto, caso um não-associado deseje comparecer ao evento, deve comprar um cartão avulso, o que lhe obriga a se deslocar até um local de venda para adquiri-lo.

\section{Tecnologia NFC}

A tecnologia NFC (Near Field Commnunication) é um padrão definido pelo "NFC Fórum", um consórcio global criado em 2004 e composto de companhias de hardware, software, cartões de crédito e bancos. Entre os principais membros do Fórum estão: Qualcomm, LG, Nokia, Huawei, HTC, Motorola, NEC, RIM, Samsung, Sony Ericsson, Toshiba, AT\&T, Sprint, Google, Microsoft, PayPal, Visa, Mastercard, American Express e Intel [NFC-Fórum, 2014].

NFC é uma tecnologia que possibilita a troca de informações sem uso de fios, utilizando radiofrequência. Uma troca de informação é estabelecida automaticamente assim que dois dispositivos estejam próximos o suficiente, sem a necessidade de configurações adicionais. Essa tecnologia teve origem no padrão RFID, mas permite um campo de atuação de até quatro centímetros, tornando-a mais vantajosa em termos de segura natroca de informações se comparada a outras, como Bluetooth. A velocidade máxima de comunicação é de $424 \mathrm{Kbps}$ [NFC-Fórum, 2014].

NFC é compatível com as infraestruturas existentes para uso de cartões sem contato, permitindo que o usuário utilize um único dispositivo em diversos sistemas. Com o uso do NFC é possível a troca de cartões de negócio, transações financeiras, acesso a informações de pôsteres inteligentes ou fornecimento de credenciais para acessar sistemas de controle com um simples toque na tela [Ceipidor, 2008].

A adoção no NFC em dispositivos móveis tem crescido rapidamente, fabricantes têm adicionado esta tecnologia nos projetos dos seus dispositivos móveis. Estima-se que já foram vendidos cerca de 400 milhões de dispositivos móveis no mundo com NFC e, para 2016, a previsão é que este número chegue a 1 bilhão [Hunter, 2013].

As principais vantagens do uso do NFC para o usuário final são: fácil conexão, transação rápida e compartilhamento de dados de forma simples [NFC-Fórum, 2014]. 


\subsection{Segurança em NFC}

Um dos fundamentos da segurança da informação está apoiado na integridade, que visa assegurar que um documento não tenha seu conteúdo alterado após ter sido assinado. Para isso, o sistema deve ser capaz de detectar alterações não autorizadas no conteúdo. O objetivo é que o destinatário verifique que os dados não foram modificados indevidamente [Schneir, 1996]. Existem várias técnicas para assegurar a integridade dos dados, uma delas se chama Hash ou Hashsum, que se baseia na criação de uma sequência de símbolos única baseada no documento ou conjunto de dados. Esta sequência é gerada a partir de um algoritmo e pode ser verificada no destinatário, desde que ele conheça o algoritmo de hash aplicado no documento [Menezes, 1996].

Outro aspecto a garantir, é o acesso à informação somente dos usuários autorizados. Uma abordagem existente é criar uma camada de autenticação na aplicação através de uma senha. No entanto, este método, de forma isolada no dispositivo móvel, poderia expor o aplicativo a softwares maliciosos, pois a senha ficaria armazenada no próprio dispositivo. Uma solução para isto é utilizar autenticação em nuvem, deixando o trabalho de validação e armazenamento de dados sensíveis do usuário em um servidor remoto [Pourghomi, 2014]. O apelo à segurança em transações de todos os tipos conduz ao desenvolvimento de sistemas com uso da tecnologia NFC, devido as suas características que nativamente promovem um nível extra de segurança nos sistemas onde ela é aplicada. No caso de substituição de cartões, ela evita "relay attacks", que são ataques que ocorrem quando a comunicação com o smartcard é retransmitida em longas distâncias através de um canal alternativo de dados [Roland, 2012]. Dentre os diversos tipos de sistemas, um deles refere-se à validação de identidade, que leva a aplicações de controle de entrada em eventos. Mesmo não sendo totalmente à prova de fraudes, conforme discutido [Madlmayr, et al.; Eun et al., 2013; Coskun et al., 2013], ele ainda é mais seguro que os cartões e ingressos impressos [Ghiron et al., 2009] e [Ceipidor et al., 2013].

Além do cumprimento da norma ISO/IEC 14443-4 [ISO, 2008], a qual padroniza o protocolo para este tipo de comunicação, outro fator importante para garantir a segurança é a curta distância necessária para haver uma comunicação, cerca de $10 \mathrm{~cm}$, permitindo que o usuário veja o terminal no qual está se comunicando [New Science, 2014]. No entanto, Ceipidor (2012), enxerga vulnerabilidades, mesmo nesta distância, então propõe o uso de algoritmos de criptografia e protocolos de autenticação entre os dispositivos.

\section{NFC Aplicado ao Controle de Entrada em Eventos}

Existem iniciativas já implantadas em alguns países, que se utilizam da tecnologia NFC para o controle de entrada de pessoas em eventos e sistemas relacionados. Um SDK disponibilizado pela controladora de cartões de crédito Visa, irá permitir que pagamentos sejam realizados diretamente pelo smartphone com NFC. Esta solução se chama "Visa payWave for Mobile e permitirá a compra de produtos no modo débito, crédito e pré-pagos, tanto em transações online quanto off-line, tendo aplicação em compra de ingressos em estádios, shows, cinemas e transportes [Visa, 2014].

No início de 2011, a BMW anunciou que estava trabalhando em um chaveiro NFC criptografado que, em conjunto com seu sistema ConnectedDrive, poderia ser usado em compras e-downloads de bilhetes de trem, ou para reserva de quartos de hotéis, sendo 
que após seu uso como chave de acesso ao quarto do hotel, também poderia ser usado no pagamento da hospedagem [Oxford, 2012].

Nos Estados unidos e no Japão, a tecnologia já é usada para comprar bilhetes de trem (Figura 2), ingressos de eventos e até para pagamento de compras. No sistema de metrô de Tóquio é possível comprar passagens apenas aproximando o celular das catracas de acesso. Esta função simples mudou a forma de viajar no país, além de aumentar a confiança na tecnologia, pois, neste caso, o usuário somente precisava fazer uma compra de pequeno valor, referente àquela viagem [Keferi, 2012].

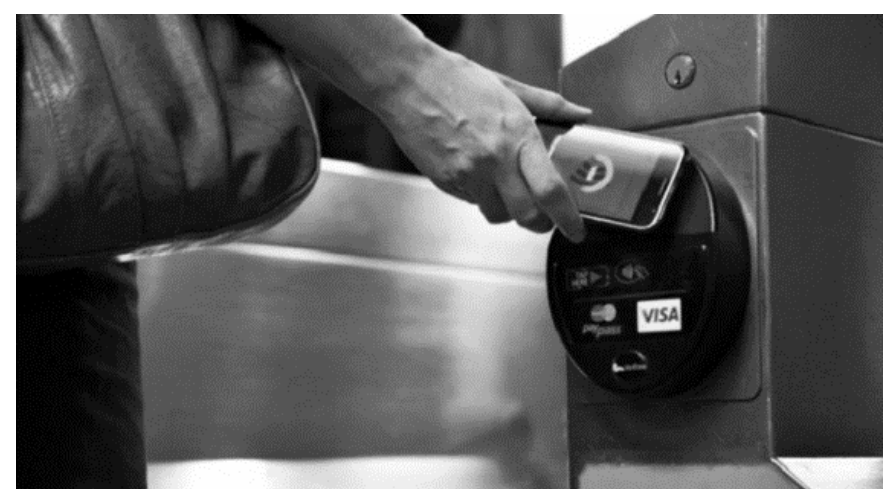

Figura 2 - Utilização do NFC para transporte ferroviário [Keferi, 2012].

Pesquisadores na Indonésia propuseram um sistema para transporte ferroviário de passageiros, utilizando smartphones com NFC em todo processo de compra do ticket e embarque, chamado de TraiNFC. O passageiro (Figura 3A) pode fazer a compra do ticket escaneando uma tag referente à viagem desejada (Figura $3 \mathrm{~B}$; Figura $3 \mathrm{C}$ ). Esta tag fica localizada dentro da estação de trem. O pagamento é realizado pela compra antecipada de créditos. No momento do embarque (Figura 3 D), o passageiro apresenta o tíquete ao fiscal através do smartphone. O fiscal na porta do trem (Figura $3 \mathrm{E}$ ), com outro smartphone, faz a conferência por NFC. Neste momento, também ocorre a invalidação do tíquete da viagem no smartphone do passageiro (Figura $3 \mathrm{~F}$ ) [Nasution, 2012].

Na França, a Air France está testando o sistema Touch\&Pass, que permitirá aos passageiros utilizem seus smartphones com NFC para se locomoverem dentro do aeroporto, desde a entrada no prédio até o embarque na aeronave (Figura 4). De acordo com a empresa, este sistema trará agilidade, facilidade e dispensará o ticket em papel, pois, além das facilidades citadas, os passageiros continuariam a usar seu tradicional sistema de check-in via smartphone [Boden, 2014].

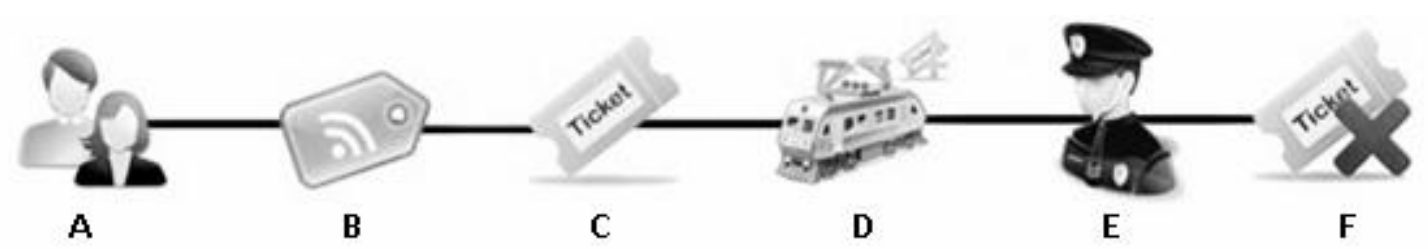

Figura 3 - Diagrama de caso de uso para transporte de passageiros. 


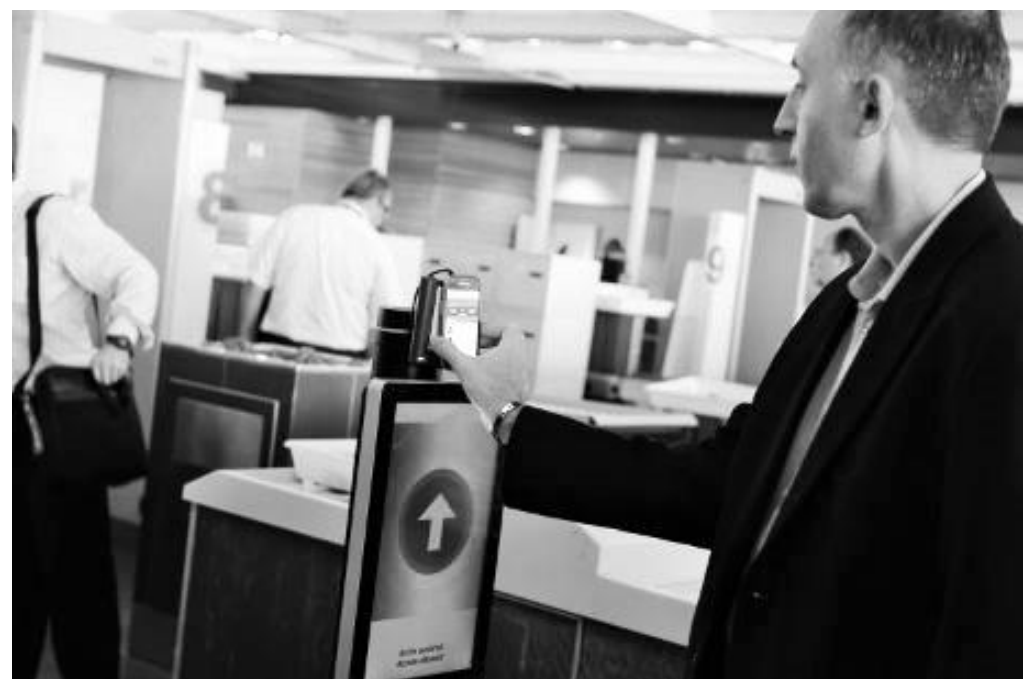

Figura 4 - Utilização de NFC para embarque aéreo na França. [Boden, 2014].

Em outro sistema, Matos (2012) desenvolveu um protótipo para dispositivos móveis Android, que interage com um sistema backend e possibilita ao produtor de evento a gestão básica para a tomada de decisões. Este sistema possibilita a consulta de eventos ativos, informando o andamento da venda de ingressos, permitindo ao produtor a virada do lote, o que significa habilitar o início de sua venda, geralmente a preços maiores. Como este sistema é dedicado aos organizadores de eventos, pode ser considerado um complemento ao proposto neste trabalho, que é dedicado aos usuários finais. Além dos sistemas descritos, outros trabalhos também analisam o uso de NFC como substituto de ingressos com em [Ghiron et al., 2009] e [Ceipidor et al., 2013].

\section{Sistema Proposto}

O Sistema proposto utiliza smartphone em conjunto com NFC, e visa permitir que o usuário adquira e utilize ingressos eletrônicos para acessar o local do evento desejado que, por sua vez, valida o acesso através do leitor NFC ligado ao Sistema de controle.

\subsection{Modelo de negócio}

A aquisição do ingresso é realizada pelo usuário através do aplicativo no smartphone, no qual exige identificação com usuário e senha. Neste aplicativo, o usuário pode procurar por opções de eventos disponíveis e adquirir o ingresso. O pagamento é realizado acessando Servidor web da empresa que organiza o evento. Agrega-se a esse Servidor o envio de mensagem de resposta indicando a efetivação da compra do ingresso.

A validação do ingresso e autorização de entrada do usuário é realizada no local do evento, através de outro dispositivo NFC conectado a um computador com acesso ao Servidor que contém as informações de ingressos válidos.

Caso o usuário distribua seus dados de acesso ao Sistema para que outra pessoa entre no evento com outro smartphone, somente o primeiro acesso é permitido pelo Servidor.

Como o acesso ao aplicativo exige usuário e senha, caso o smartphone seja furtado ou perdido, um terceiro de posse do smartphone teria que descobrir os dados de acesso para 
utilizar o ingresso, mesmo assim, o usuário lesado pode retirar um ingresso físico após devida identificação em um guichê no evento. Neste caso o Sistema invalida o ingresso. No caso de falha no Servidor de Acesso, é possível manter um Servidor redundante que assuma a operação sem prejuízos maiores para o andamento do evento.

\subsection{Arquitetura do Sistema}

A Figura 5 ilustra a arquitetura do Sistema proposto. O protótipo desenvolvido para o usuário final é composto por um smartphone Nokia Lumia 720 com Windows Phone versão 8.1 e NFC compatível. A parte do sistema de gerência, estabelecida no local do evento, é composta por um computador com Windows 8.1 e leitor NFC modelo "Sony USB NFC Reader RC-S380”.

No computador, é executado o aplicativo desenvolvido para Windows, que é responsável pela validação do ingresso e liberação da entrada do usuário, através do acesso ao Webservice no Servidor.

O Servidor é o responsável por armazenar o banco de dados MySQL e o WebService Apache para comunicação com o computador e com o smartphone, ambos por Internet.

Tanto o aplicativo para Smartphone quanto o para Desktop foram desenvolvidos sobre o Visual Studio Ultimate 2013, utilizando SDK para Windows 8.1 e Windows Phone 8.1.

O Diagrama de Componentes da Figura 6 representa todos os submódulos do Sistema e a comunicação hierárquica entre eles.

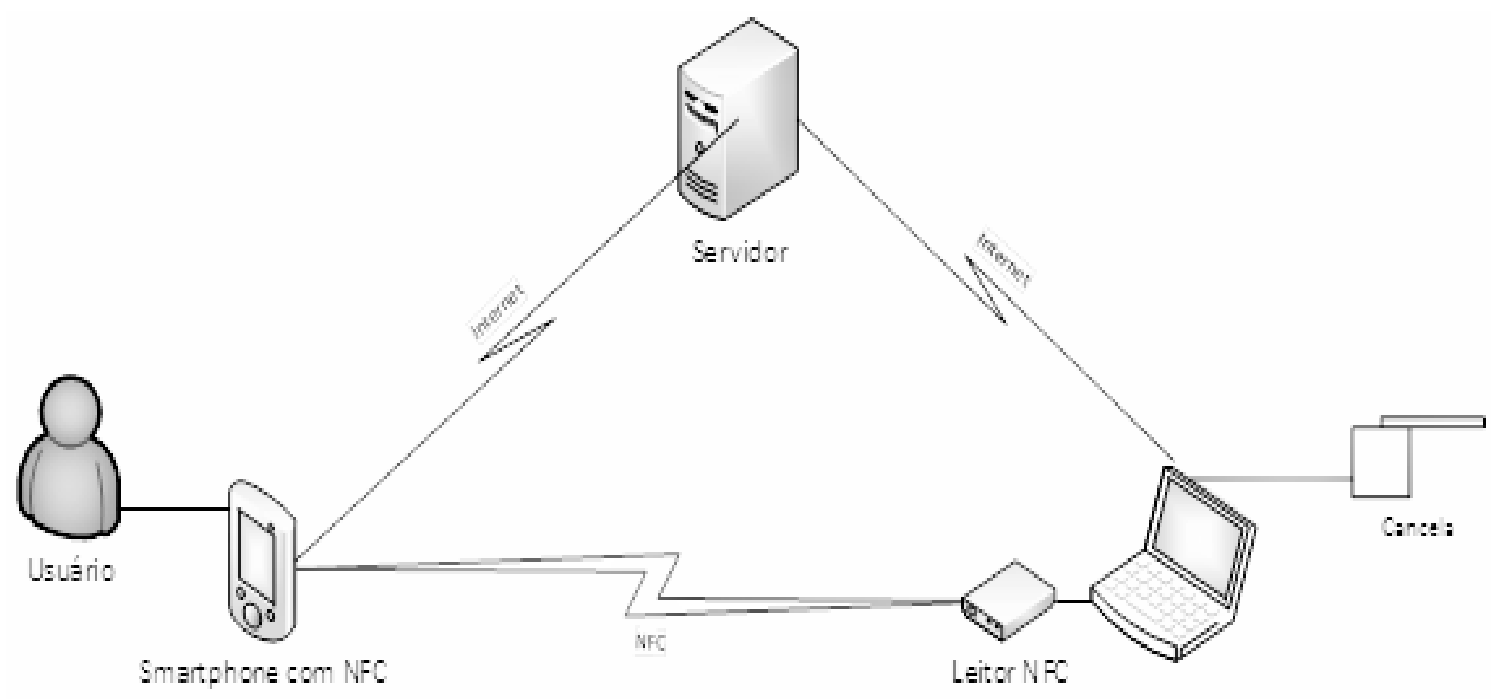

Figura 5 - Representação da arquitetura do Sistema proposto. 


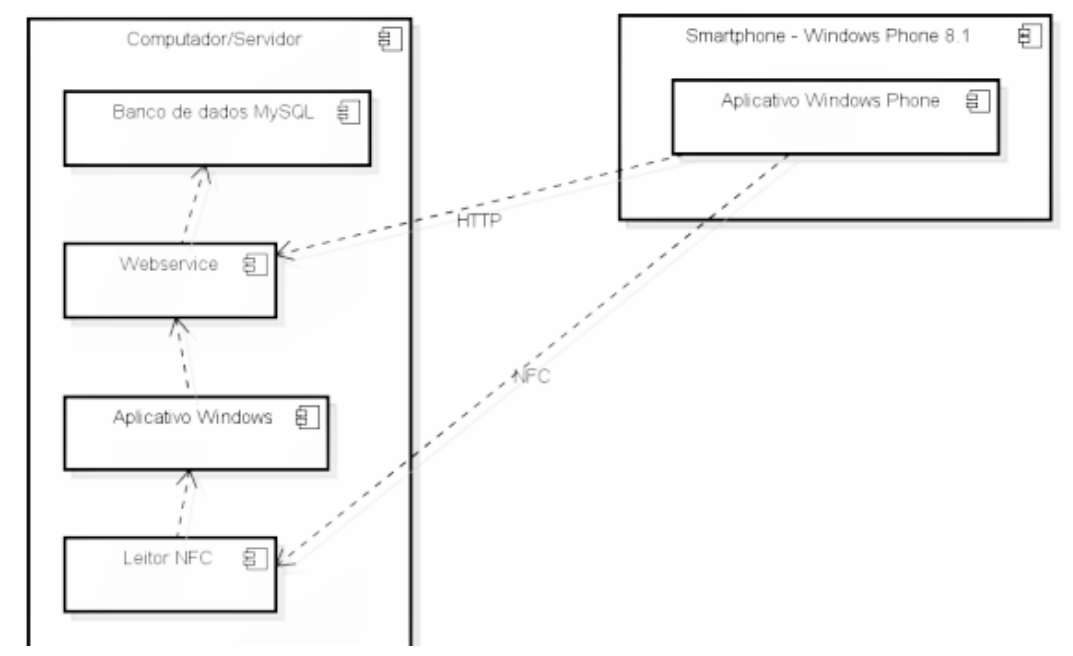

Figura 6 - Diagrama de componentes: Representação da arquitetura do protótipo.

A estrutura do banco de dados central (Servidor), que armazena todos os dados do Sistema é apresentada na Figura 7. Ela é simples, mas suficiente para perfeito funcionamento do Sistema.

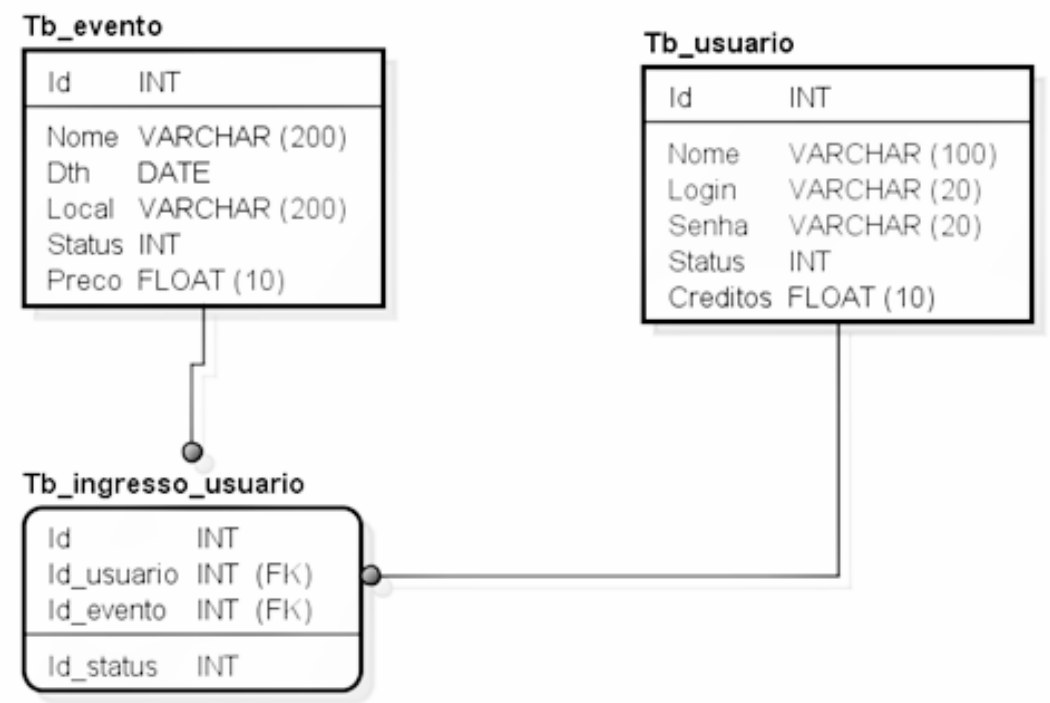

Figura 7 - Estrutura do banco de dados do Servidor.

\subsection{Fluxo de Uso de Telas de Usuário}

Inicialmente, o usuário se conecta informando seu usuário e senha para ter acesso à tela de opções: "Ingressos ativos", "Loja", "Ingressos utilizados" e "Login/Logoff". O Diagrama de Estados da Figura 8 mostra o fluxo de navegação entre as telas. As telas têm fluxos de navegação direto, o que garante poucas operações até a conclusão de cada operação. Todas as operações partem de um menu principal. A operação mais simples é a de consulta dos ingressos utilizados. A compra de ingressos tem duas etapas composta da escolha do ingresso e a confirmação dessa escolha. A mais complexa é a de uso do ingresso. Nela o usuário seleciona o ingresso na lista de ingressos válidos e confirma seu uso. Depois, aproxima o smartphone do Sistema de controle de acesso que habilita 
ou nega a entrada do usuário. Mesmo envolvendo três etapas o processo é rápido, não exigindo operações complexas por parte do usuário. Normalmente as duas primeiras etapas do uso do ingresso são disparadas antes do acesso ao local do evento, por exemplo, na fila de entrada do evento, sobrando apenas a validação final no momento do acesso. Esse processo tem dois impactos principais: o tempo reduzido de acesso do usuário e a boa aceitação de uso do Sistema, pois o único tempo requisitado é na validação junto a catraca de acesso. A busca por usabilidade tem como objetivos apresentar um sistema comercialmente viável, de baixo custo de desenvolvimento e infraestrutura, fácil operação e principalmente que permita avaliação da aceitação da tecnologia NFC na sociedade brasileira atual.

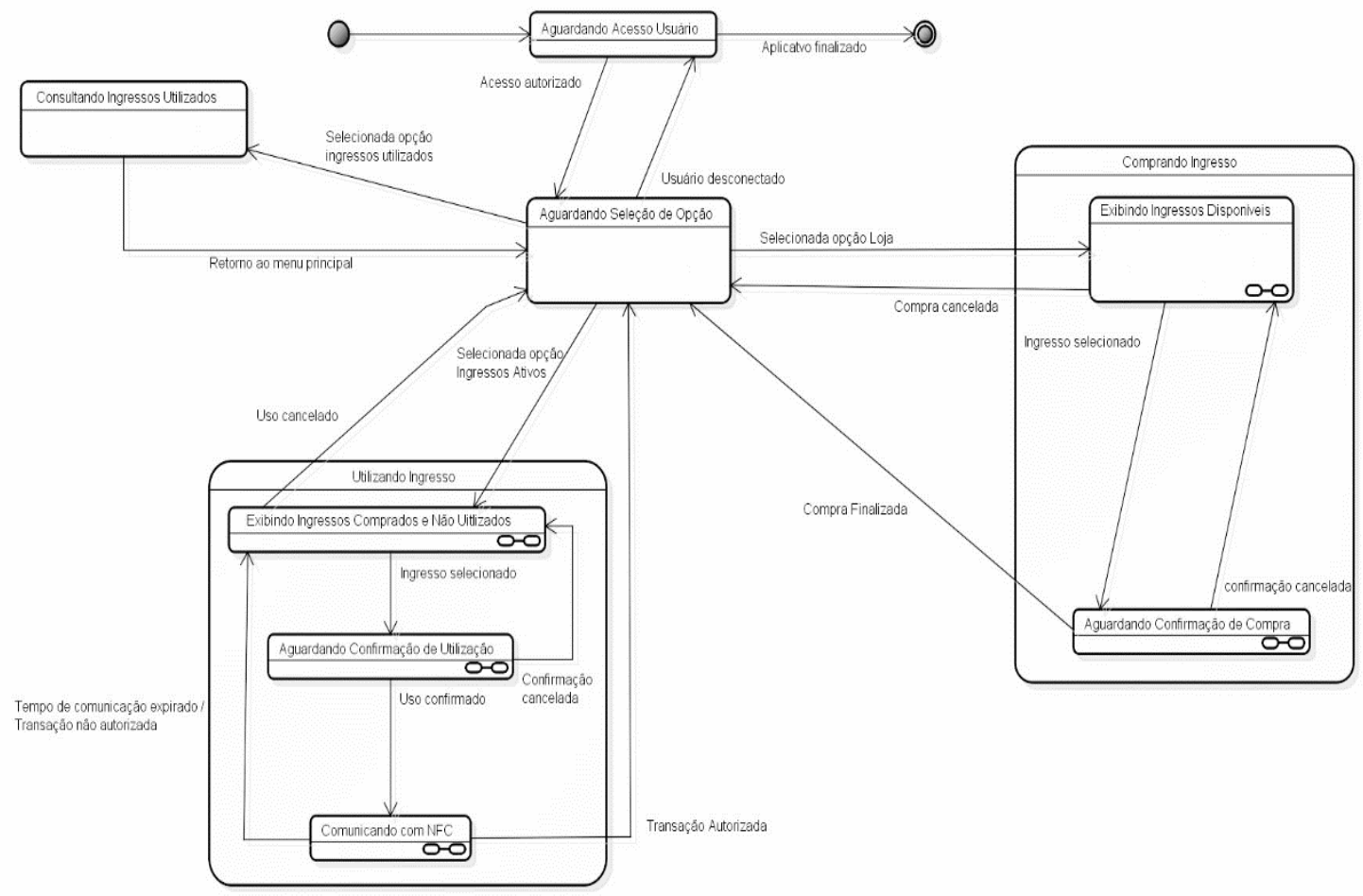

Figura 8 - Diagrama de Estados do aplicativo de usuário.

\subsection{Características do Protótipo}

A liberação da entrada do usuário é indicada visualmente na tela do aplicativo Desktop, não há um mecanismo externo, como uma cancela.

Neste protótipo, é utilizado um único computador com todos componentes necessários, isto é, aplicativo Windows, leitor NFC, banco de dados e Webservice. Desta forma, o aplicativo Windows acessa diretamente o Webservice, através do endereço localhost da própria máquina. Também, para acesso ao WebService pelo smartphone, é utilizado um roteador, criando uma rede local. Portanto, os testes realizados com este protótipo, não utilizaram acesso à Internet.

Tanto o cadastro de novos usuários, quanto o cadastro de ingressos à venda são realizados diretamente no banco de dados. Não foi elaborado um sistema de pagamento. Por se tratar de um protótipo, não foram implementados controles importantes de 
segurança, como criptografia, e integridade. No entanto, foi inserida autenticação de usuário com senha. Estas simplificações, típicas de protótipos, foram assumidas por se entender que elas não afetam de forma significativa os testes com usuários, quando avaliados facilidade de uso do Sistema e tempo de acesso. Assume-se que o tempo de comunicação entre o computador e a cancela é desprezível, pois os fabricantes disponibilizam interfaces Serial RS232, RS485, TCP/IP 10/10 ou 10/100 para comunicação, conforme constatado com fabricantes [K2Sistemas, 2015; Orped, 2015; RWTech, 2015]. Mesmo em rede TCP/IP, o que poderia ser origem de atrasos, a comunicação pode ser feita via intranet, portanto com tempo de resposta mínimo, não afetando o acionamento da cancela.

Conforme apresentado na Figura 6, o Sistema foi construído com o Servidor, autoriza o acesso do usuário, conectado via internet. Este é um possível ponto de atraso e fragilidade que pode afetar dois requisitos importantes de sistemas, a confiabilidade e a disponibilidade [Pradhan, 1996]. Porém, devido a característica de modularidade do Sistema, esse inconveniente pode ser evitado instalando o Servidor na intranet do local. As vantagens do uso de intranet são o aumento significativo da confiabilidade e disponibilidade do Sistema e a diminuição de seu tempo de resposta. As desvantagens são o aumento de custos, pois cada evento tem que prover mais pessoas especializadas prontas para atuar em caso de falha, em muitos locais a infraestrutura não provê uma intranet, então deve-se instalar uma. Caso a comunicação se faça via wireless, todos os cuidados com segurança devem ser observados a fim de evitar ataques.

As adaptações inseridas no protótipo para emular a realidade não alteram seu comportamento. Todo o processo desde a aquisição do ingresso até a entrada no evento é completo, ativando todos os módulos do Sistema proposto, conforme pensado em uma aplicação real.

\section{Experimentos e Análise de Resultados}

Os experimentos buscam coletar as impressões de usuários que utilizaram o Sistema em ambiente controlado. Ao todo, quinze pessoas foram entrevistadas após o uso do Sistema. As faixa-etárias dos entrevistados envolvem diferentes perfis: 3 pessoas $(20 \%)$ com idades até 29 anos, 6 pessoas (40\%) entre 30 e 49 anos e 6 pessoas (40\%) com idade acima de 49 anos.De acordo com várias pesquisas de perfil social [Morris, 2000], [Agarwal, 2009], [Erkki, 2013], [Niehaves, 2014], cada pessoa enxerga e consegue utilizar novas tecnologias com diferentes graus de dificuldade,então, uma amostragem com grupos de pessoas de diferentes faixas etárias, também tende a dar informações mais precisas sobre a aceitação das pessoas a novas tecnologias.

Seguiu-se rigorosamente o mesmo procedimento para todos os envolvidos. Primeiramente, foi explicado o objetivo do trabalho, tecnologias envolvidas, assim como todos os componentes do protótipo. Após, foi demonstrado na prática o funcionamento do Sistema, desde a aquisição do ingresso até sua utilização para acessar o evento. Em seguida, cada participante foi convidado a utilizar o aplicativo, realizando a escolha, aquisição e uso de ingresso. Por fim, cada um respondeu um questionário com perguntas incluindo diversos aspectos relacionados ao Sistema proposto. Em nenhum momento houve intervenção externa, a Tabela 1 mostra as questões aplicadas aos usuários. A Questão 1 utilizou escala de razão baseada na idade, enquanto as 
Questões 2 a 7 foram fundamentadas na escala Likert, com 4 escalas homogêneas [Chisnall, 1973].

Tabela 1. Perguntas aplicadas aos usuários do Sistema proposto.

\begin{tabular}{|c|c|c|}
\hline$\#$ & Pergunta & Respostas Válidas \\
\hline 1 & Qual a sua faixa etária de idade? & $\begin{array}{l}\text { Até } 29 \text { anos. } \\
\text { Entre } 30 \text { e } 49 \text { anos. } \\
\text { Mais de } 49 \text { anos. }\end{array}$ \\
\hline 2 & $\begin{array}{l}\text { Você conhecia a tecnologia NFC (Near Field } \\
\text { Communication)? }\end{array}$ & $\begin{array}{l}\text { Sim, sabia do que se tratava. } \\
\text { Sim, mas não conhecia profundamente. } \\
\text { Muito pouco. } \\
\text { Não conhecia. }\end{array}$ \\
\hline 3 & $\begin{array}{l}\text { O Sistema apresentado é um ótimo substituto a } \\
\text { ingressos tradicionais. }\end{array}$ & \multirow{5}{*}{$\begin{array}{l}\text { Discordo totalmente. } \\
\text { Discordo parcialmente. } \\
\text { Concordo parcialmente. } \\
\text { Concordo totalmente. }\end{array}$} \\
\hline 4 & O Sistema apresentado é confiável. & \\
\hline 5 & A aplicação é fácil de ser utilizada. & \\
\hline 6 & $\begin{array}{l}\text { A aplicação facilita a compra e utilização de } \\
\text { ingressos. }\end{array}$ & \\
\hline 7 & $\begin{array}{l}\text { A tecnologia NFC poderia ser utilizada em } \\
\text { diversas outras aplicações. }\end{array}$ & \\
\hline
\end{tabular}

A Figura 8 mostra o nível de conhecimento sobre NFC dos entrevistados, nota-se que a grande maioria tem pouco conhecimento a respeito do tema. Mesmo em uma amostra pequena, este é um indica que no país poucas pessoas estão familiarizadas com o tema.

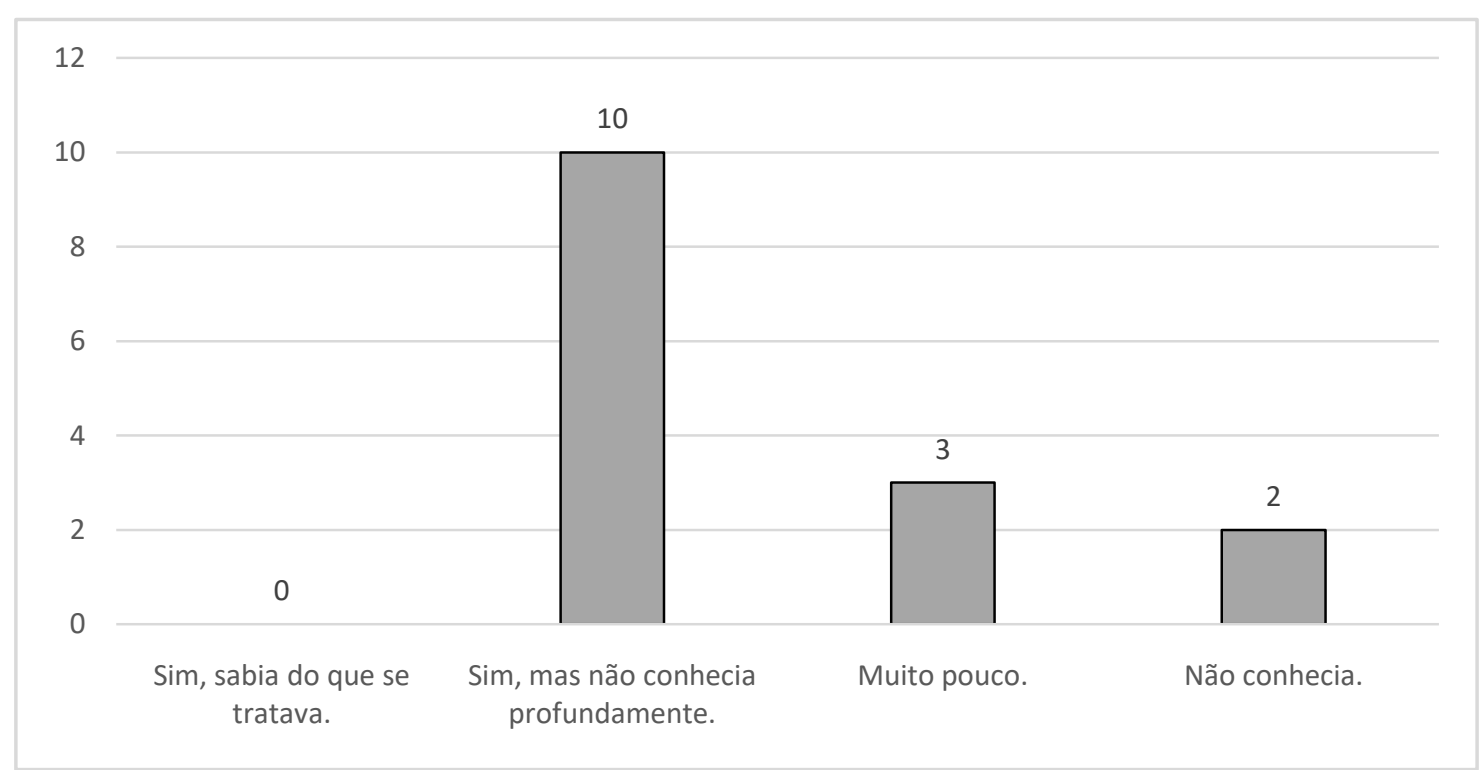

Figura 8. Resposta à Pergunta 2 da Tabela 1. 
A Tabela 2 mostra os resultados da pesquisa para as outras perguntas do questionário. A Pergunta 3 identifica a aceitação dos usuários a tecnologia NFC, a Pergunta 4 refere-se confiabilidade, as Perguntas 5 e 6 focam na experiência de uso, enquanto a Pergunta 7 identifica a aceitação da tecnologia NFC para outros tipos de sistema. Observa-se que em todas as questões o Sistema proposto e a tecnologia NFC são aceitos, com algumas variações para mais ou menos positivas. Mesmo junto ao público acima de 49 anos, que conhecidamente é mais resistente a novas tecnologias, não houve rejeições extremas.

Tabela 2. Resultados das respostas dos entrevistados as Perguntas 3 a 7 da Tabela 1.

\begin{tabular}{|c|c|c|c|c|}
\hline \# da Pergunta & $\begin{array}{c}\text { Discordo } \\
\text { totalmente }\end{array}$ & $\begin{array}{c}\text { Discordo } \\
\text { parcialmente }\end{array}$ & $\begin{array}{c}\text { Concordo } \\
\text { parcialmente }\end{array}$ & $\begin{array}{c}\text { Concordo } \\
\text { totalmente }\end{array}$ \\
\hline 3 & $0 \%$ & $0 \%$ & $13 \%$ & $87 \%$ \\
\hline 4 & $0 \%$ & $7 \%$ & $36 \%$ & $57 \%$ \\
\hline 5 & $0 \%$ & $0 \%$ & $13 \%$ & $87 \%$ \\
\hline 6 & $0 \%$ & $0 \%$ & $13 \%$ & $87 \%$ \\
\hline 7 & $0 \%$ & $0 \%$ & $13 \%$ & $97 \%$ \\
\hline
\end{tabular}

Observou-se que todos os entrevistados utilizaram de forma rápida e com facilidade o Sistema. Embora a tecnologia NFC corrobore na facilidade de uso, a aprovação no quesito usabilidade está muito mais atrelada ao modelo simplificado de uso que, com poucos toques no smartphone, concede acesso ao evento.

O Sistema tem outras potenciais características, na medida que o ingresso está ligado ao smartphone, é possível rastrear com maior facilidade quem entrou no estádio e a que horas. Essas informações são importantes para a organização do evento. Além disso, no momento da saída do local do evento, ou em caso de pânico, é possível enviar mensagens aos smartphones direcionando as pessoas de forma inteligente para as saídas, evitando que elas se concentrem em algumas saídas e assim, diminuindo o tempo de evacuação do local.

\section{Conclusão}

Este trabalho apresentou o uso da tecnologia NFC aplicado a um sistema de aquisição e controle de entrada de ingressos. Um dos focos do trabalho foi avaliar a aceitação da tecnologia NFC por parte de usuários brasileiros. O outro foco do trabalho foi apresentar um modelo de controle de entrada em eventos guiado pela simplicidade de uso por parte dos usuários e de fácil implementação e aplicação. O modelo de controle de ingressos foi implementado e testado por usuários em ambiente controlado. Um questionário foi aplicado aos usuários com intuito de avaliar o nível de aceitação, confiabilidade e usabilidade do mesmo.

Os resultados mostram que o modelo proposto e a tecnologia NFC são bem aceitos em todos os quesitos. Devido a amostra de usuários avaliados ter sido pequena, a aceitação observada deve ser vista apenas como um indício de que a tecnologia NFC tenha boa aceitação no mercado brasileiro para controle de entrada de pessoas em eventos. 
Como trabalhos futuros deve-se ampliar a aplicação da pesquisa a mais usuários para comprovar a tendência de aceitação do Sistema e da tecnologia NFC. Para esta segunda avaliação será melhorado o Sistema, adicionado o processo de pagamento e permitir que o usuário se localize dentro do local do evento através de um mapa e tags NFC distribuídas em pontos estratégicos. Pretende-se ainda realizar um teste de campo com o Sistema e ampliá-lo para outras aplicações com: compra e utilização de cupons de restaurantes em sites de compra coletiva, que geralmente são muito burocráticos e requerem a impressão em papel do cupom e sistemas de pagamento direto entre consumidor e vendedor, avaliando assim a aceitação de usuários brasileiros à tecnologia NFC em outras áreas de aplicação.

\section{Referências}

Agarwal, R., et. al., Social Interactions and the "Digital Divide": Explaining Variations in Internet Use, V.20, Issue 2, 2005.

Boden, R. Air France lets passengers board planes with NFC, NFC World+. Disponível em http://www.nfcworld.com/2014/07/02/330100/air-france-lets-passengers-boardplanes-nfc/. Acesso em 07 de Julho de 2014.

Boulos, M., Wheeler, S., Tavares, C., Jones, R., How smartphones are changing the face of mobile and participatory healthcare: an overview, with example from eCAALYX, BioMedical Engineering OnLine, Vol. 10, 2011.

Ceipidor, U. B., et al., NFC: Integration between RFID and Mobile, state of the art and future developments, Emerging Technologies for Radiofrequency Identification, p.78-81, 2008.

Ceipidor, U.B., Medaglia, C.M., Marino, A., Morena, M., Sposato, S., Moroni, A., Di Rollo, P.,Morgia, M.La, Mobile ticketing with NFC management for transport companies. Problems and solutions, 5th International Workshop on Near Field Communication (NFC), p.1-6, 2013.

Ceipidor, U. B., et al., KerNeeS: A protocol for mutual authentication between NFC phones and POS terminals for secure payment transactions. 9th International ISC Conference on Information Security and Cryptology (ISCISC), ISBN 978-1-46732387-1. 2012.

Cesca, C. G., Organização de eventos: manual para planejamento e execução. 9a. Ed. São Paulo: Summus, ISBN 978-85-323-0401-8. 2008.

Chisnall, P., Marketing Research: Analysis and Measurement. McGraw-Hill, 1973.

Coskun V., Ozdenizci B., Ok K., A Survey on Near Field Communication (NFC) Technology, Wireless Personal Communications: An International Journal, v.71 n.3, p.2259-2294, 2013.

Erkki, K., The Effect of Age on Technology Acceptance among Field Police Officers, Proceedings of the 10th International ISCRAM, Germany, 2013.

EunH., Lee H., Oh H., Conditional privacy preserving security protocol for NFC applications, IEEE Transactions on Consumer Electronics, Vol.59 Issue 1, 2013.

Galitz, W.O., The essential guide to user interface design: an introduction to GUI design principles and techniques, John Wiley \& Sons, Inc., $3^{\mathrm{a}}$ ed., 2007. 
Gavinho, L., Ingresso do futuro, Guia Ingresse, Disponível em: $<$ http://guia.ingresse.com/2013/07/ingressos-do-futuro/>, Acesso em 21 de Maio de 2014.

Ghiron, S. L., Sposato, S., Medaglia, C. M., Moroni, A., Nfc ticketing: A prototype and usability test of an nfc-based virtual ticketing application. International Workshop on Near Field Communication, p.45-50, 2009.

Hix, D., Hartson, H. R., Developing User Interfaces: Ensuring Usability Through Product \& Process, John Wiley \& Sons, Inc.,1993.

Hunter, P., The time for NFC is now. NFC Fórum. 04 de novembro de 2013. Disponível em: <http://nfc-forum.org/post-in-blog-test-2/>. Acesso em 15 de Abril de 2014.

IDC, Worldwide Smartphone 2014-2018 Forecast and Analysis. Disponível em: $<$ http://www.idc.com/getdoc.jsp?containerId=247140>. Acesso em 14 de Abril de 2014.

Igoe, T, Jepson, B., Beginning NFC: near field communication with Arduino, Android, \&Phonegap", O’Reilly Media, Inc., p.11-15, 2014.

ISO, Identification cards - Contactless integrated circuit cards - Proximity cards Part 4: Transmission protocol: ISO/IEC 14443-4, 2008.

K2Sistemas, Disponível em: <http://www.k2seguranca.com.br/solucao/controle-deacesso $>$. Acesso em 06 de Outubro de 2015.

Keally, M.,et al., PBN: Towards Practical Activity Recognition Using Smartphonebased Body Sensor Networks, Proceedings of the 9th ACM Conference on Embedded Networked Sensor Systems, p.246-259, 2011.

Keferi, M., Near-Field Communication Is Shifting Marketing in Japan, 12 Jun. 2012. Disponível em: <http://adage.com/article/global-news/field-communication-shiftingmarketing-japan/235260/>. Acesso em 15 de Abril de 2014.

Madlmayr G., Langer J., Kantner C., Scharinger, J., NFC Devices: Security and Privacy, Third International Conference on Availability, Reliability and Security, ARES 08. 2008.

Matos, A. V., Desenvolvimento de um protótipo de aplicação para um dispositivo com sistema operacional Android para a gestão de um evento por um produtor. Universidade Tecnológica Federal do Paraná, 2012. Dissertação (Pós-graduação para Especialização em Tecnologia Java). Disponível em $<$ http://repositorio.roca.utfpr.edu.br/jspui/bitstream/1/830/1/CT_JAVA_VII_2012_1 9.PDF>. Acesso em 14 de Outubro de 2014.

Menezes, A. J., Oorschot, P. C. van; Vastone, S., A. Handbook of Applied Cryptography, $5^{\text {a }}$ ed., p.321-376, 1996.

Michahelles, F.Thiesse, F., Schmidt, A., Williams, J. R., Pervasive RFID and Near Field Communication Technology, IEEE Computer Society, Pervasive computing, 2007.

Moriya, T. M., GIR, E.,Hayashida, M., Escala de Atitude Franteá AIDS: Uma análise psicométrica. Revista Latino-Americana de Enfermagem. Vol. 2. Num. 2. p. 37-53. 
jul. 1994. Disponível em <http://www.scielo.br/pdf/rlae/v2n2/v2n2a04.pdf $>$ Acesso em 16 de Setembro de 2013.

Morris, M. G., Venkatesh, V., Age Differences in Technology Adoption Decisions: Implications for a Changing Work Force. Personnel Psychology, V.53, p.375-403, 2000 .

Nasution, S. M., et al. Prototype of Train Ticketing Application Using Near Field Communication (NFC) Technology on Android Device, International Conference on System Engineering and Technology (ICSET), 2012.

New Science. Transaction Security: Mobile Payment Security: Ble or NFC. p. 7, 2014. $<$ newscience.ul.com/wp-content/uploads/2014/07/NS_TS_Article_MobilePaymentS ecurity.pdf $>$ Acesso em 01 de Novembro de 2015.

Niehaves, B., Plattfaut, R., Internet adoption by the elderly, European Journal of Information Systems, V.23, n.6, p.708-726, 2014.

NFC-Fórum. NFC and Contactless Technologies. Disponível em: <http://nfcforum.org/what-is-nfc/about-the-technology/>. Acesso em 15 de Abril de 2014.

Orped, Disponível em: <http://www.ortep.com.br/?produtos=pedestal-inox $>$. Acesso em 06 de Outubro de 2015.

Oulasvirta, A., Rattenbury, T., Ma L., Raita E., Habits make smartphone use more pervasive, Personal and Ubiquitous Computing, Vol. 16, Issue 1, pp 105-114, 2012.

Oxford, T., O toque mágico. The Review. N. 1. P. 13. 2012. Disponível em: $<$ https://www.gemalto.com/brochures/download/review_feb12/GEMALTO_winter_ 2012 po.pdf $>$. Acesso em 15 de Abril de 2014.

Pourghomi, P., Saeed, M. Q., Guinea, G., A SecureCloud-based NFC Mobile PaymentProtocol. Disponível em $<$ https://eprint.iacr.org/2014/538.pdf $>$. Acesso em 08 de Outubro de 2014.

Pradhan, D. K., Fault-Tolerant System Design. Prentice Hall, New Jersey, 1996.

Roland, M., Software Card Emulation in NFC-enabled Mobile Phones: Great Advantage or Security Nightmare?, Fourth International Workshop on Security and Privacy in Spontaneous Interaction and Mobile Phone Use (IWSSI/SPMU), Newcastle, UK, 2012.

RWTech, Disponível em: <http://www.rwtech.com.br/produtos.php?cat=2>. Acesso em 06 de Outubro de 2015.

Teleco,Mercado mundial de smartphones. Disponível em: $<$ www.teleco.com.br/smartphone.asp > . Acesso em 04 de Outubro de 2015.

Schneir, B., Applied Cryptography, 2ªed., Mountain View, p.14-15, 1996.

Souza, R., Conhecimentos bancários, Editora Áudio Ltda., Edição 2013, 2013.

Visa, Visa payWave for Mobile, Disponível em: <developer.visa.com/paywavemobile>. Acesso em 14 de Abril de 2014. 